\title{
Trends in lung cancer emergency presentation in England, 2006-2013: is there a pattern by general practice?
}

Camille Maringe ${ }^{1 *} \mathbb{D}$, Nora Pashayan², Francisco Javier Rubio', George Ploubidis ${ }^{3}$, Stephen W. Duffy ${ }^{4}$, Bernard Rachet ${ }^{1}$ and Rosalind Raine ${ }^{2}$

\begin{abstract}
Background: Emergency presentations (EP) represent over a third of all lung cancer admissions in England. Such presentations usually reflect late stage disease and are associated with poor survival. General practitioners (GPs) act as gate-keepers to secondary care and so we sought to understand the association between GP practice characteristics and lung cancer EP.

Methods: Data on general practice characteristics were extracted for all practices in England from the Quality Outcomes Framework, the Health and Social Care Information Centre, the GP Patient Survey, the Cancer Commissioning Toolkit and the area deprivation score for each practice. After linking these data to lung cancer patient registrations in 2006-2013, we explored trends in three types of EP, patient-led, GP-led and 'other', by general practice characteristics and by socio-demographic characteristics of patients.

Results: Overall proportions of lung cancer EP decreased from 37.9\% in 2006 to 34.3\% in 2013. Proportions of GPled EP nearly halved during this period, from 28.3 to $16.3 \%$, whilst patient-led emergency presentations rose from 62.1 to $66.7 \%$. When focusing on practice-specific levels of EP, $14 \%$ of general practices had higher than expected proportions of EP at least once in 2006-13, but there was no evidence of clustering of patients within practice, meaning that none of the practice characteristics examined explained differing proportions of EP by practice.

Conclusion: We found that the high proportion of lung cancer EP is not the result of a few practices with very abnormal patterns of EP, but of a large number of practices susceptible to reaching high proportions of EP. This suggests a system-wide issue, rather than problems with specific practices. High proportions of lung cancer EP are mainly the result of patient-initiated attendances in A\&E. Our results demonstrate that interventions to encourage patients not to bypass primary care must be system wide rather than targeted at specific practices.
\end{abstract}

Keywords: Emergency presentation, Lung cancer, General practice

\section{What this paper adds?}

- What is already known on the subject

Over a third of lung cancer patients are diagnosed as emergencies.

The emergency route to diagnosis is sub-optimal, associated with late-stage diagnosis and poor survival.

\footnotetext{
* Correspondence: camille.maringe@lshtm.ac.uk

${ }^{1}$ Cancer Survival Group, London School of Hygiene and Tropical Medicine,

Keppel street, London WC1E 7HT, UK

Full list of author information is available at the end of the article
}

- What this study adds

The study finds that most emergency presentations reflect that patients by-pass primary care.

In the period 2006-2013, close to $15 \%$ of practices show higher than expected proportions of emergency presentation.

There are no General Practice characteristics predictive of unexpectedly high or low levels of emergency presentation.

(c) The Author(s). 2018 Open Access This article is distributed under the terms of the Creative Commons Attribution 4.0 International License (http://creativecommons.org/licenses/by/4.0/), which permits unrestricted use, distribution, and reproduction in any medium, provided you give appropriate credit to the original author(s) and the source, provide a link to the Creative Commons license, and indicate if changes were made. The Creative Commons Public Domain Dedication waiver (http://creativecommons.org/publicdomain/zero/1.0/) applies to the data made available in this article, unless otherwise stated. 


\section{Background}

New diagnoses of cancer through emergency hospital presentation are often related to delayed diagnosis [1]. In England, they represent almost a quarter of new cancer diagnoses [2]. Patients diagnosed with cancer through emergency presentation usually have advanced tumour stage [3] and lower one-year survival than those presenting via other routes [2, 4]. Improving early diagnosis of cancer was a priority of the Cancer Reform Strategy [5] and is now part of the six strategic priorities of the 2015-20 Strategy for England [6].

Delay in diagnosis can occur at patient, primary care, and/or secondary care levels [7, 8]. For example, delays may occur when a patient does not recognise cancer symptoms or seek health care, when a healthcare practitioner misinterprets the symptoms, or does not investigate or refer the patient for further investigation, or when there is long waiting time to be seen by a specialist, leading to delay in initiation of the appropriate treatment.

In addition to patient and doctor delays, the organisational structure of healthcare systems influences care seeking. A qualitative study from Denmark hypothesised that the role of general practitioners (GPs) as gatekeepers to the rest of the healthcare system and providing continuity in doctor-patient relationship may influence care seeking decisions [9]. The UK and Denmark, which both have comprehensive gatekeeper and list systems, have significantly lower one-year relative survival from cancer than countries such as Sweden or Canada, which have less stringent gatekeeper and list systems $[9,10]$.

Over 30,000 people are diagnosed with lung cancer each year in England. Lung cancer remains the leading cause of cancer deaths in England in both men and women [11]: one-year net survival is $33.2 \%$ in men and $38.9 \%$ in women, and 5-year net survival is as low as 11 . $1 \%$ in men and $15 \%$ in women [12].

There is no national screening programme to identify lung cancer at an early stage in the UK. However, unlike most other common cancers, patients can be investigated in primary care by chest X-ray, which means that general practitioners have access to an additional diagnostic test. The National Institute for Health and Care Excellence (NICE) guidelines for referral or request of chest X-ray are based on unexplained and persistent symptoms or signs such as cough, weight loss, hoarseness, etc. [13]. Despite the availability of diagnostic investigation in primary care, a high proportion of lung cancer patients are still not referred according to the recommended and most straightforward route to a respiratory clinic for diagnosis [14].

A retrospective analysis of Hospital Episode Statistics (HES) between 1999 and 2006 showed that 52\% of patients with lung cancer in England were admitted as emergencies. Such admissions were more common in women, older patients and patients from deprived areas [15]. In 2007, it was estimated using routine data (cancer registry, HES and National Cancer Waiting Times $(\mathrm{NCWT})$ ), that $38 \%$ of patients in England were diagnosed with lung cancer through emergency presentation [2]. Despite small improvements in recent years [16], late diagnosis and emergency presentation remain a major concern in lung cancer.

The reasons for delay in diagnosis and emergency presentation are complex and multi-factorial. Patients characteristics (sex, age, deprivation, place of residence) $[17,18]$ and cancer awareness may influence timeliness of presentation. Nevertheless, primary care health professionals have an important role in early diagnosis and there have been calls to better understand the primary care factors associated with emergency presentations [19] as well as their regional variations [18].

\section{Aim and objectives}

We aimed to describe and explain the heterogeneity in proportions of lung cancer diagnoses through emergency presentation - thereby referred to as proportions of EP between practices in 2006-2013. First, we explored the variability in the national proportions of three types of emergency presentations over time. Then, we depicted the variation in proportions of emergency presentation by practice. Finally, we explored the association between practice characteristics and proportions of emergency presentations adjusting for variations by patient characteristics.

\section{Methods \\ Material \\ Information on cancer patients}

In England, 264,813 patients were diagnosed and registered in the population-based National Cancer Registry between 2006 and 2013 with an invasive primary malignancy of the lung. We linked these individual records to the Lung Cancer Audit Data (LUCADA) and the Cancer Analysis System (CAS) data to enhance information on stage at diagnosis. Together, these datasets provided information on patient's characteristics (code of the registered practice, date of birth, sex, postcode of usual address, vital status, date of last vital status) and tumour characteristics (date of diagnosis, stage, histology, morphology, site). Deprivation is measured at the Lower Super Output Area (LSOA) level, using the Index for Multiple Deprivation (IMD) income domain. The IMD scores are ranked and split according to quintiles, thereby dividing the LSOAs in five groups of increasing deprivation. Patients are allocated to a deprivation group given their LSOA of residence at the time of diagnosis. A validated algorithm that makes use of stage-related variables present in these datasets was applied to derive Tumour, Nodes, Metastasis 
(TNM) stage at diagnosis [20]. Stage at diagnosis remained missing for $31.8 \%$ of lung cancer patients (ranging between $62.9 \%$ in 2006 and $8.7 \%$ in 2013).

The route to diagnosis variable defining the emergency presentation status of each patient is derived using information from Hospital Episode Statistics and other data sources [21]. Not only does this variable provide information on one of eight possible routes to diagnosis (death certificate only registration, emergency presentation, GP referral, inpatient elective, other outpatient, two-week wait, unknown and screening), it also provides information on the point of contact that initiated the route to the diagnosis.

\section{Information on general practices}

We gathered data items about General Practices from the following publicly available data sources:

a. The Quality Outcomes Framework (QOF) indicators, from the Quality Management and Analysis System (QMAS) database from NHS Digital, including data from April 2010 to March 2011 from practices in England [22]. It is the annual reward and incentive programme detailing GP practice achievement results. We selected indicators from the clinical (Chronic Obstructive Pulmonary Disease-COPD- and Respiratory) and organisational domains most closely related to lung cancer.

b. Individual items about each General Practice, from NHS Digital: the registered patient list with breakdown by age category and sex, the number of GPs, the number of GPs per practice population (as of 30 September 2010), proportions of GPs qualified in the UK and average age of GPs per practice (as of 30 September 2011).

c. The Index for Multiple Deprivation 2010 (IMD) of each practice, provided by the Public Health England's Knowledge and Intelligence Team on behalf of the Department of Health. This is estimated by taking a weighted average of the IMD scores for each Lower Super Output Area (LSOA) in which a given practice has registrations.

d. All items from the GP Patient Survey (GPPS), except the items relative to NHS dentistry (section K), collected between April 2010 and March 2011 [23]. The GPPS gather patients' feedbacks about their experiences of their GP surgery.

e. Items from the General Practice Profiles (PP), downloaded from the Cancer Commissioning Toolkit (CCT) in July 2013, containing information on four domains: demographics, cancer screening, cancer waiting times, presentation and diagnostics [24]. These represent data on cancer services at GP level.
The items identified from each of these data sources are shown in Additional file 1: Table S1.

Due to the timeframe of the General Practices data captured, we matched the information only to the patients diagnosed in 2010. After excluding 2916 patients $(8.7 \%)$ who did not get matched to any practice-level information due to missing or erroneous code of practice, the analyses of the association between GP Practice characteristics and EP included 33,468 patients.

\section{Statistical methods}

Trends in emergency presentation, association with patient characteristics

We examined the changing distributions of EP, and its two main sub-types (patient- and GP-led), by year of diagnosis. We defined patient-led emergencies (i.e. patients who bypassed primary care) as "Accident and emergency (A\&E) or dental casualty department of the Health Care Provider", and GP-led emergencies as "General practitioner: after a request for immediate admission has been made direct to a Hospital Provider (i.e. not through a Bed bureau), by a general practitioner or deputy". All other emergency types (Emergency: via Bed Bureau, including the Central Bureau; Emergency: via consultant outpatient clinic; Emergency: other means, including patients who arrive via the A\&E department of another healthcare provider; Other, undefined start points; Following an emergency admission; Referral from an accident and emergency department; Following an accident and emergency attendance) are referred to as "Other".

\section{Proportion of emergency presentation by GP practice}

We used funnel plots [25] to display the practicespecific proportions of EP, and highlight practices with higher proportions than expected. The proportions of EP were plotted against a measure of their precision, i.e. the number of lung cancer patients diagnosed in each practice. The funnels around the pre-defined target, set as the national proportion of emergency presentation for patients with a valid practice number in that year, represent confidence limits at 99.7 and 95\% (3 and 2 standard deviations, respectively). We flagged the practices with proportions of EP outside the 95\% confidence limit in 2010, and tracked their performance over the years 2006-2013 to see whether they were habitual outliers.

\section{Association between GP practice characteristics and EP}

Exploratory and confirmatory factor analyses were used to reduce the dimension of the general practice information (GPPS, QOF and PP datasets) to meaningful factors or latent variables. More precisely, we ran specific analyses for each dataset (GPPS, QOF and PP) and the factors identified were used to summarise the data and reduce dimensionality. They were then entered in a multilevel structural 
equation model with a logistic link. We used the software Mplus [26].

In order to investigate whether GPs' and practices' characteristics predict emergency diagnosis of lung cancer, we aimed to model practice-specific clustering. Patients who attend a given practice will be affected by the same practice-level characteristics. We took account of this cluster using mixed logistic regression models incorporating random intercepts associated to the practice. The variance of that random intercept reflects how much of the overall variation in EP is explained by the practice-level characteristics. The need for including random intercepts was assessed in terms of the percentage of variance explained by these variables (variance partition coefficient, VPC), and the Akaike Information Criterion (AIC) compared to that of models without random intercepts.

The outcome of each logistic model was EP status at patient level, and the models were adjusted for variables from different practice-level datasets as well as patient-level variables such as age and deprivation. We believe stage at diagnosis lies in the causal pathway between patients or practice characteristics and EP, thus, we do not present any models that include adjustment for stage at diagnosis; rather we compare the results of stage-specific models.

Variable selection techniques were employed to identify the relevant features, from the practice-specific information available, that impact EP. These include stepwise AIC variable selection, Lasso and Elastic-Net methods [27], and significance assessment. All models were adjusted for patient characteristics known to be associated with EP (sex, age and deprivation).

The $\mathrm{R}$ software was used to perform the logistic regressions and report the various statistics.

\section{Results}

Variability in national proportions of EP Trends in emergency presentation

The overall proportion of emergency presentation, for lung cancer patients diagnosed in 2013, was $34.3 \%$, compared to $37.9 \%$ for patients diagnosed in 2006 (Table 1). Whilst patient-led emergencies increased from $62.1 \%$ of all emergencies in 2006 to $66.7 \%$ in 2013, there was a marked continuous statistically significant decrease in GP-led emergencies from $28.3 \%$ in 2006 to $16.3 \%$ in 2013 of all emergencies (Table 1).

The number of lung cancer patients increased by 13 . 7\% between 2006 and 2013 (from 30,879 to 35,097), leading to a net increase of $2.9 \%$ in the number of emergency diagnoses (from 11,690 to 12,028); Table 1. The increase in the absolute numbers (+603/year) of lung cancer patients was mostly absorbed through non-EP GP referral routes which increased (+546/year) while the numbers of GP-led EP decreased (-193/year).
However, there was also a non-negligible increase in the numbers of patient-led EP (+109/year) and other EP (+132/year, Table 1$)$.

\section{Patient characteristics and emergency presentation}

High proportions of lung cancer EP were strongly associated with living in more deprived areas and late or missing stage, and to a lesser extend with being female (Table 2 and web-Additional file 2: Table S2). Although the overall proportions of EP decreased, these patterns remained over the whole study period. Because stage information was almost complete in 2013, the stage-related pattern was clearer than in 2006 and showed higher EP proportions among more advanced disease (Table 2).

The temporal shift between sub-types of EP was similar across the deprivation categories, with the exception of the most deprived where most of the decrease in GPled EP was transferred to the 'Other' EP type (Table 2 and Fig. 1). Patients from more deprived backgrounds showed higher proportions of patient-led emergency presentation than patients from the least deprived backgrounds $(p=0.02$, Table 2$)$.

\section{Proportion of emergency presentation by GP practice}

Figure 2 shows a series of funnel plots describing the heterogeneity in practice-specific proportions of EP for the years 2006 to 2013. It highlights where practices stand with respect to their proportions of EP of lung cancer patients in the corresponding year, by their number of new cases of lung cancer as a measure of precision of the estimates. For a given year, very few practices had proportions of EP above the upper 95\% confidence limit: for example, in 2010, this included only 150 practices out of 7514 (red triangles in Fig. 2). However, in the same calendar year, half of the practices (3667) presented a maximum of three lung cancer patients, which means that setting the EP proportion at the extreme level of $0 \%$ in the 150 upper outliers would decrease the national level of EP by only $2 \%$. Furthermore, practicelevel proportions changed dramatically year on year because of the high number of practices with few patients: cumulatively, as many as 1163 General Practices in England, i.e. $14.6 \%$ of the total number of General Practices, fell above the upper limit of the funnel plots at least once between 2006 and 2013.

\section{Association between GP practice characteristics and EP}

Explanatory and confirmatory factor analyses reduced the practice-level datasets to only two or three factors for each of the different data sources. The factors could be labelled "Trust and confidence in the nurse" and "Trust and confidence in the GP" from the GPPS data, "Diagnosis of cancer", "Two-Week Wait referrals" and "Use of screening" from the PP data, and "COPD" and 


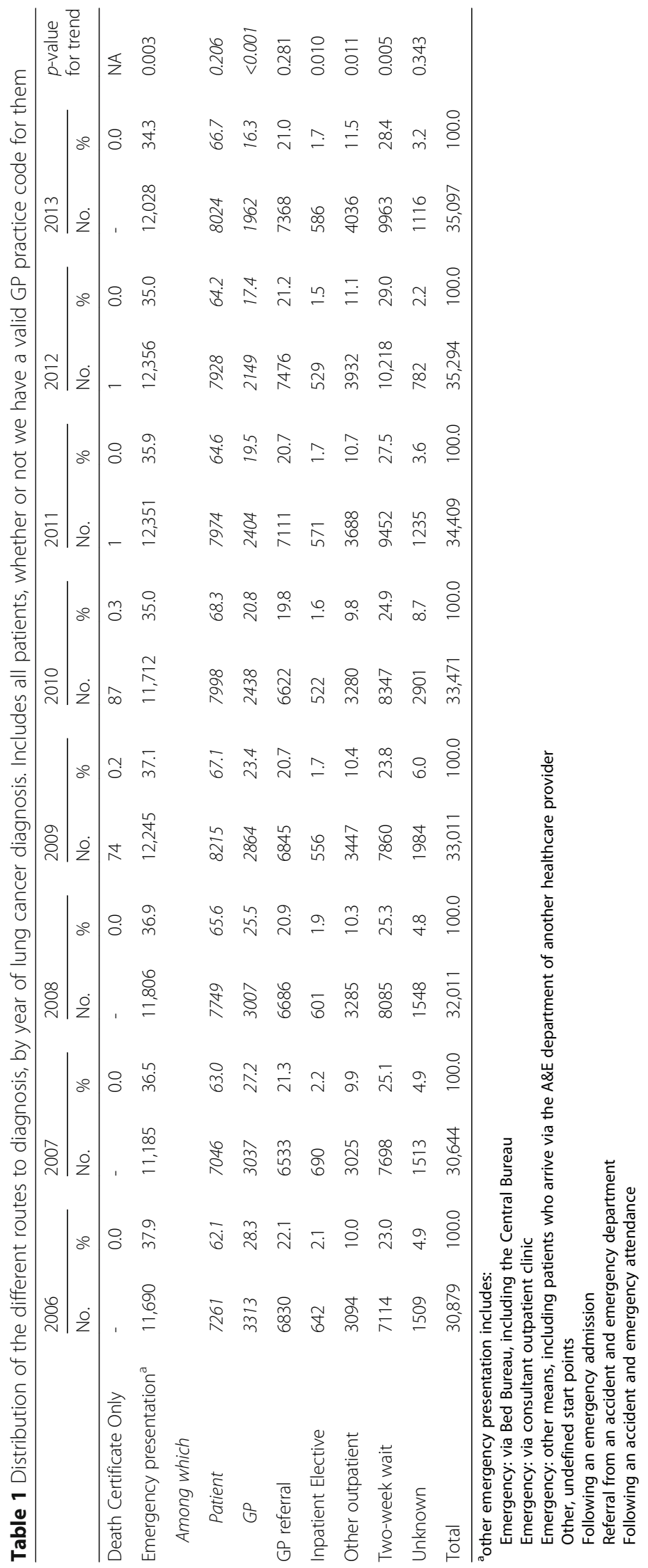


Table 2 Proportions of EP, and sub-types of EP by patients characteristics for patients diagnosed in 2006 and 2013

\begin{tabular}{|c|c|c|c|c|c|c|c|c|}
\hline & \multicolumn{4}{|l|}{2006} & \multicolumn{4}{|l|}{2013} \\
\hline & \multirow{2}{*}{$\begin{array}{l}\text { Proportions } \\
\text { with EP }\end{array}$} & \multicolumn{3}{|c|}{ Among EP patients } & \multirow{2}{*}{$\begin{array}{l}\text { Proportions } \\
\text { with EP }\end{array}$} & \multicolumn{3}{|c|}{ Among EP patients } \\
\hline & & GP-led & Patient-led & Other & & GP-led & Patient-led & Other \\
\hline All patients & 37.9 & 28.3 & 62.1 & 9.5 & 34.3 & 17.0 & 36.4 & 100.0 \\
\hline \multicolumn{9}{|l|}{ Sex } \\
\hline men & 37.1 & 28.1 & 62.4 & 9.6 & 33.7 & 16.1 & 67.0 & 16.9 \\
\hline women & 38.9 & 28.7 & 61.8 & 9.5 & 34.9 & 16.6 & 66.3 & 17.1 \\
\hline \multicolumn{9}{|l|}{ Deprivation } \\
\hline Least deprived & 33.4 & 32.3 & 59.0 & 8.7 & 30.6 & 18.3 & 64.9 & 16.7 \\
\hline dep 2 & 35.2 & 32.5 & 59.1 & 8.4 & 33.6 & 18.7 & 64.6 & 16.7 \\
\hline dep 3 & 37.5 & 30.6 & 61.1 & 8.4 & 34.5 & 17.6 & 66.5 & 15.9 \\
\hline dep 4 & 39.3 & 27.7 & 61.8 & 10.5 & 35.3 & 16.1 & 67.7 & 16.1 \\
\hline Most deprived & 40.9 & 23.3 & 66.1 & 10.6 & 35.6 & 12.9 & 68.1 & 18.9 \\
\hline$p$-value for trend & $<0.001$ & 0.02 & 0.02 & 0.09 & 0.03 & 0.04 & 0.02 & 0.39 \\
\hline \multicolumn{9}{|c|}{ TNM stage at diagnosis } \\
\hline stage I & 16.4 & 21.3 & 58.3 & 20.4 & 17.5 & 11.0 & 52.6 & 36.4 \\
\hline stage II & 14.4 & 26.3 & 53.7 & 20.0 & 18.3 & 10.4 & 58.3 & 31.3 \\
\hline stage III & 24.4 & 27.0 & 59.5 & 13.5 & 23.0 & 14.0 & 63.5 & 22.5 \\
\hline stage IV & 41.7 & 31.6 & 60.3 & 8.1 & 43.8 & 17.5 & 68.6 & 13.9 \\
\hline$p$-value for trend & 0.11 & 0.03 & 0.48 & 0.04 & 0.13 & 0.08 & $<0.001$ & $<0.001$ \\
\hline missing stage & 41.1 & 27.6 & 63.2 & 9.2 & 51.0 & 18.0 & 72.0 & 10.0 \\
\hline
\end{tabular}

"Smoking services" from the QOF data. The few factors retained were not predictive of emergency presentation (Odds Ratio, OR, close to 1, data not shown).

Additional file 3: Table S3 indicates that there was no evidence of practice-level clustering of EP. In fact, the VPC was extremely small in most cases, around $1 \%$, and the AIC also favoured models without random intercept. Consequently, all conclusions were based on logistic regression models. All variable selection techniques led to similar conclusions that most practice-specific variables were not associated to EP. The stage-specific analysis identified a few practice characteristics which were weakly associated with lung cancer EP. These were: getting through to the practice on phone, ability to see a doctor in next two days or more than two days after last tried, how good the doctor is at asking about symptoms, how good the nurse is at giving enough time. The odds ratios (ORs) between each predictor and emergency presentation were not significant and with wide confidence intervals (see Additional file 4: Table S4). The addition of variables to the model did not improve its predictive performance. In Additional file 5: Figure S1 we provide the ROC curves and the corresponding Cindex, which illustrate the prediction ability of these models. Comparing panels A and B, the practice-level variables did not improve the predictive performance of the models: C-index increased by only $1 \%$, which was irrelevant for practical purposes. We obtained the same conclusions using the Brier score, which represents an alternative measure of predictive accuracy.

\section{Discussion}

The proportion of lung cancer presenting as an emergency decreased slightly in England between 2006 and 2013 and was accompanied by a steep drop in GP-led emergency referrals. By 2013, two thirds of emergency presentations were patient led, of whom $27 \%$ were in the most deprived quintile and $73 \%$ were late stage (Additional file 2: Table S2). There was no consistency with respect to the characteristics of general practices which exhibit high proportions of EP: for each year between 2006 and 2013, a different set of only 5\% of practices had higher than expected levels of EP. This sheds some light as to why we cannot build a practice profile predictive of EP and demonstrates the importance of a system wide rather than targeted approach to reducing emergency presentations.

\section{Trends in sub-types of EP}

Although the proportion of lung cancer emergency presentations decreased slightly over the time period examined, the number of patients diagnosed every year increased, resulting in a stable number of emergency cases per year (Table 1). GPs' role as gate keepers for secondary care aims to enhance the appropriateness of 


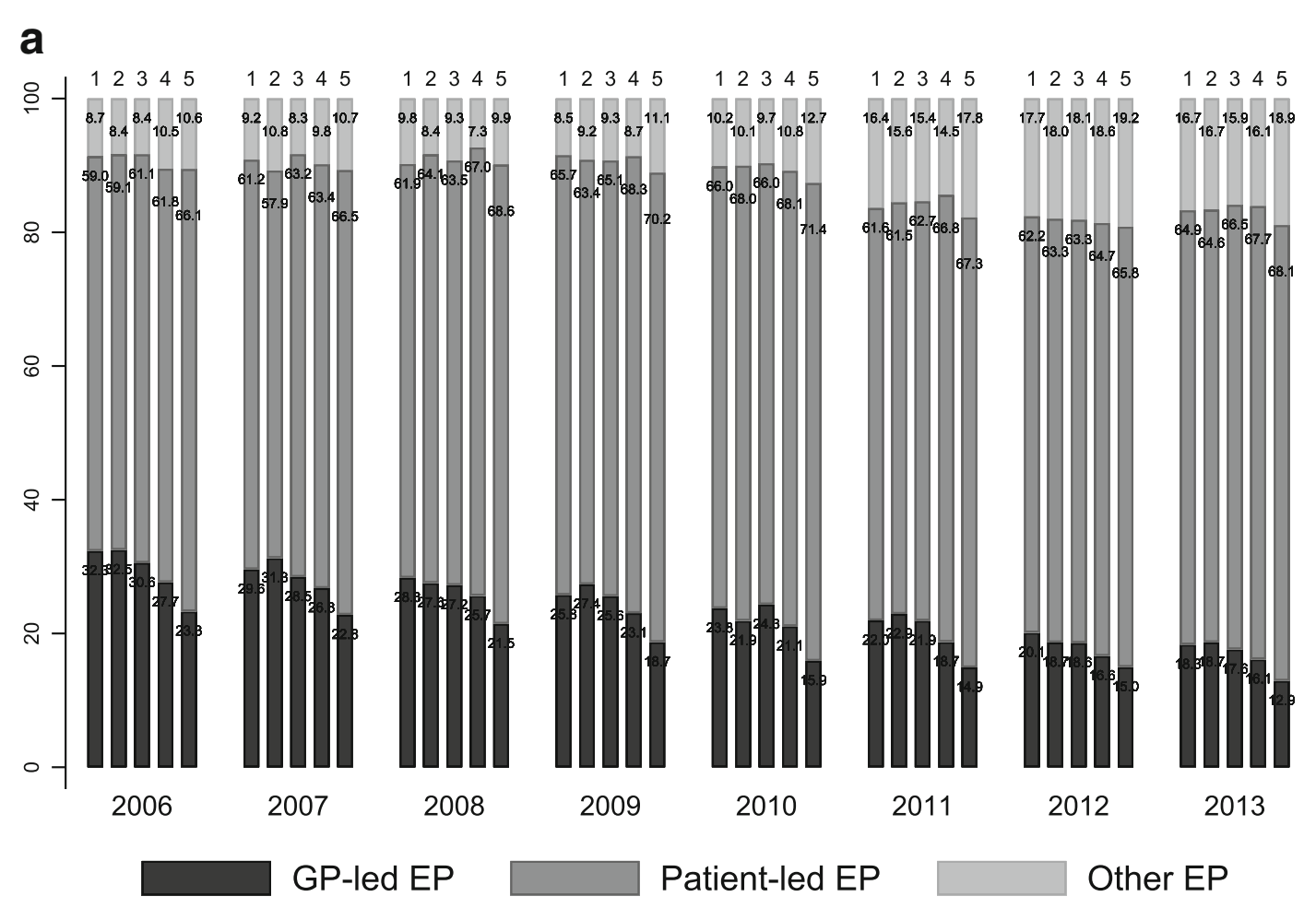

1: Least deprived, ... 5: Most deprived

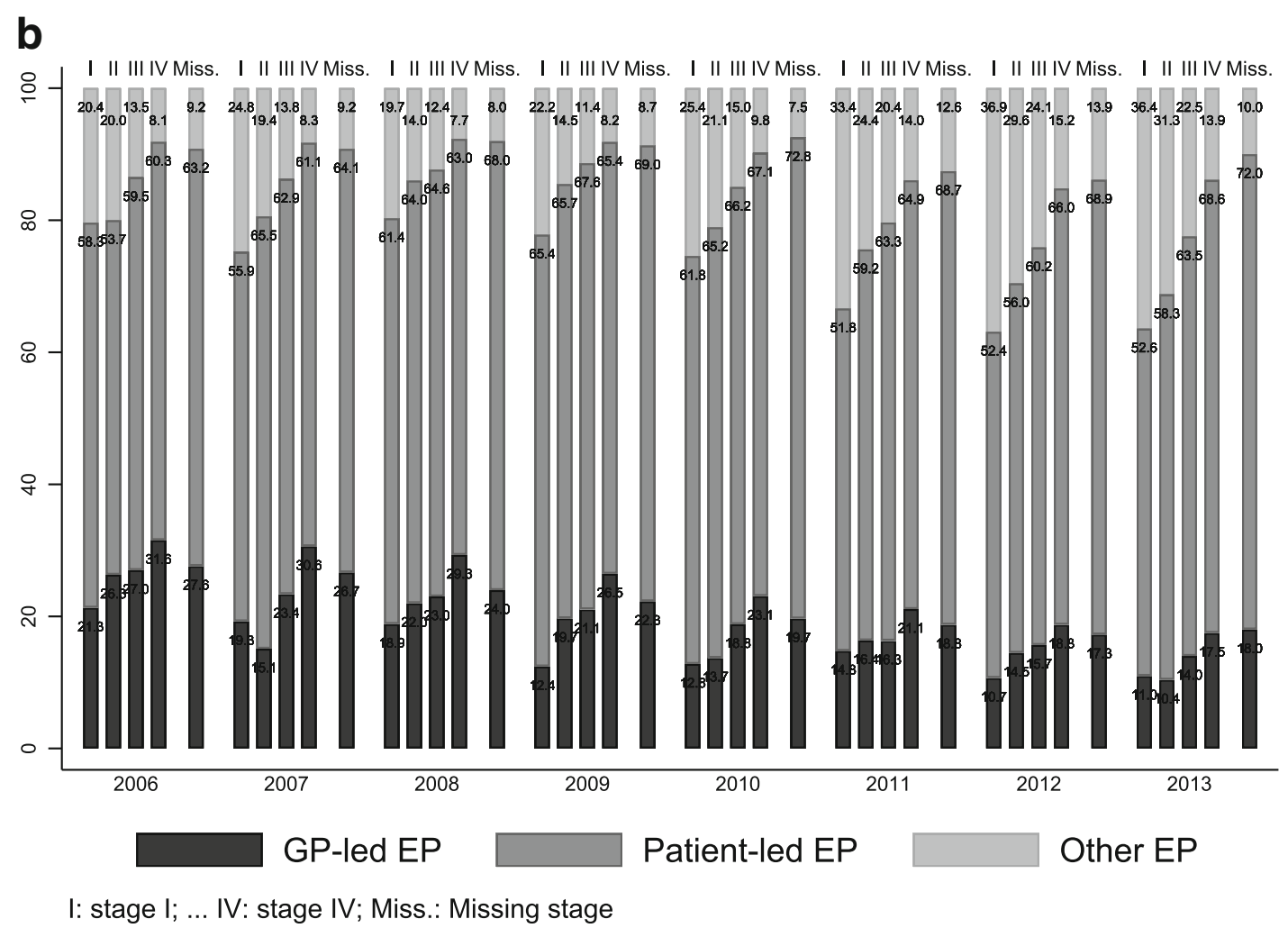

Fig. 1 Types of emergency presentation, by deprivation (a) and stage at diagnosis (b), lung cancer patients diagnosed in 2006 to 2013 


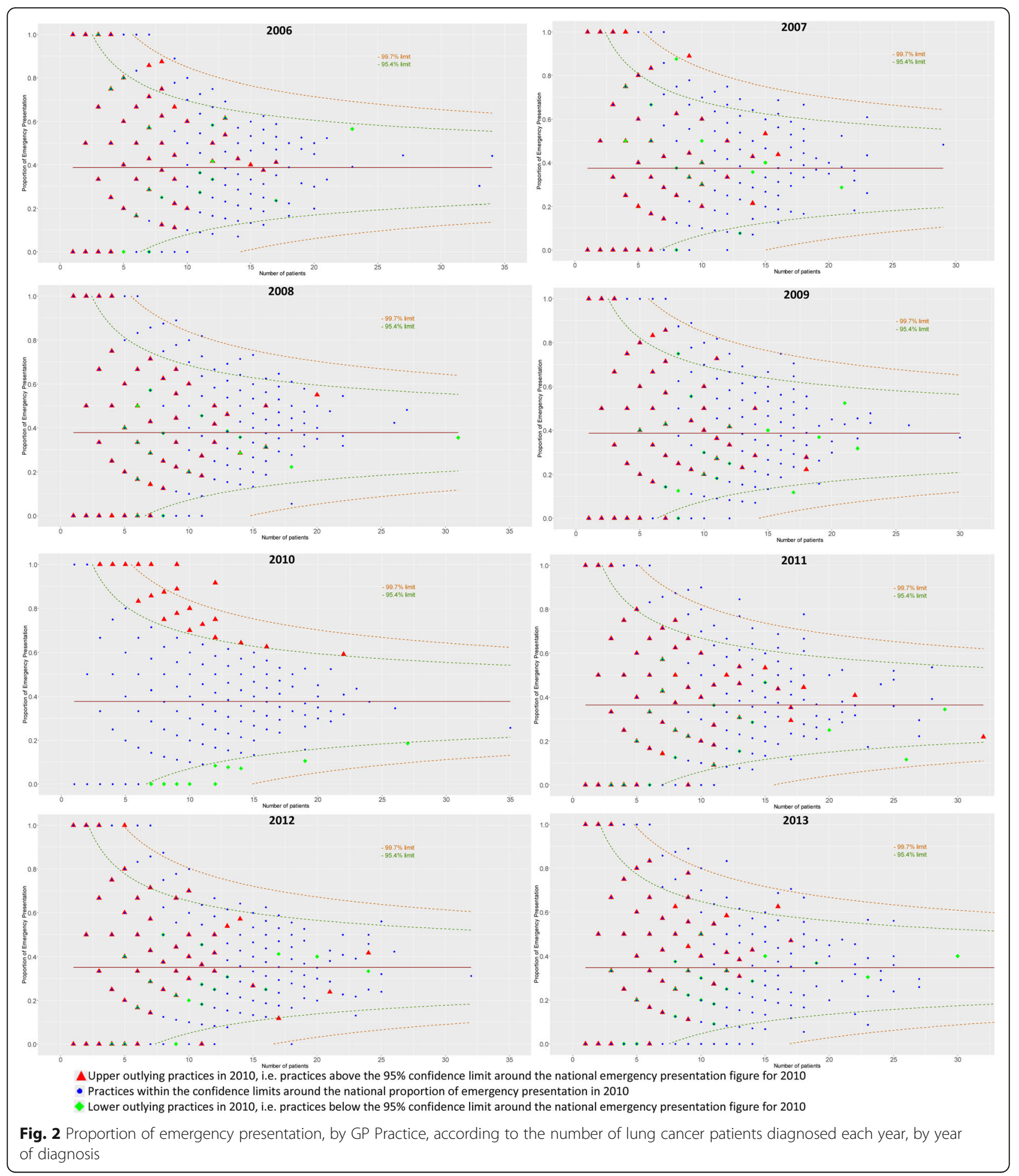

setting for patient care and to reduce unnecessary pressure on secondary care [28]. Nonetheless our results suggest that two-thirds of patients presenting as EP by-pass primary care. A better understanding is needed about the motivations and the previous primary care pathway that led these patients to access care via A\&E. This high proportion, combined with (a) the concomitant decrease in GP-led EP proportion and increase in patient-led EP proportion, and (b) the increasing proportion of EP from A\&E of another healthcare provider, is noteworthy. Our results cannot be attributed to any change in definition of these 
sub-types of EP, because definitions did not change between 2006 and 2013 .

We also show that the least deprived patients are more likely to be referred by their GP to A\&E compared to the most deprived. The extent to which this might be driven by more frequent GP attendance, higher levels of health literacy or other factors could not be explored in this analysis.

\section{Patterns of EP by practice and practice characteristics}

We found that the high proportion of EP is not the result of a few practices with very abnormal patterns of EP. Rather, it is the combined effect of the great majority of practices with proportions of EP around an already high national average. An illustration of the extent of this problem is that, to reduce the national average of EP from 37.6 to 30\% in 2010, 662 practices with observed highest proportions of EP (9\% of all practices diagnosing lung cancer patients that year) would need to have a proportion of EP of $0 \%$. This numerical example illustrates that a targeted intervention on a few practices [29] may have little effect on the national proportions. Furthermore, the targeted practices would change every year.

Previous research identifies practice characteristics associated with increased EP. These include poorer access to general practice, measured as the proportion of patients who were able to obtain an appointment on their last attempt [30], and lower proportions of patients who had confidence and trust in their doctor [31], and discontinuity in consultation [32]. However, none of these analyses include an evaluation of the predictive performance of the selected model and variables, which reduces the utility of the findings. Our research suggests that none of the GP practice characteristics available for national-level analysis satisfactorily predict EP. Our results are in line with a review of 22 studies investigating EP in lung and colorectal cancer patients, concluding that no study found clear evidence between primary care factors and EP [33]. Similarly in 2001, it was established that emergency admission of colorectal cancer was not associated with present aspects of primary health care organization [34].

A recent review on the evidence about emergency presentations [35] highlights that, although the mechanisms leading to EPs are not fully understood, there is still a substantial proportion of avoidable emergency presentations. Avoidable EPs are hypothesised to result from several successive or independent "omissions", on the part of the patients and GPs, with respect to their actions towards signs and symptoms of cancer [36]. Patients may lack knowledge of cancer symptoms, or delay seeking health advice or investigations [37]. Nation-wide campaigns such as "Be clear on cancer" aim to tackle these causes of delay. In addition, primary care practitioners may overlook cancer symptoms, delay tests, investigations or referrals to secondary care [38]. This may in part be associated with GPs and patients prioritising other complaints: some EPs are the result of the combination of several factors, including the presence of other diseases [39].

\section{Strengths and limitations}

To our knowledge, this study is the most comprehensive analysis on the associations between the general practice characteristics and EP. We linked administrative and survey data to records of cancer patients from the wellestablished population-based Cancer Registry for England. We adopted different analytical approaches to investigate those associations, i.e. exploratory and confirmatory structural equation modelling approach, as well as two model selection strategies. We finally looked at the predictive performance of the selected models and factors.

However our research is limited by our lack of information on the extent of primary care involvement in 'patientled' attendances. This meant that we were unable to estimate the proportion of patients coded as patient-led EP who were sent to A\&E by their GPs. These patients may explain some of the rise in patient-led EP, and the sudden increase from 2011 in proportions of patients referred to $A \& E$ via "the A\&E department of another healthcare provider". Furthermore, previous research which linked CPRD (Clinical Practice Data Link) to cancer registrations for colorectal cancer [40] showed that although primary care use and access was similar between patients with and without EP of their colon cancer, EP patients were less likely to have red-flag symptoms recorded in primary care in the year prior to the diagnosis.

GP practices only see a limited number of lung cancer patients every year, leading to high variability in their proportions of EP. Nonetheless the methods used in this paper to detect associations have a good power since the sample sizes remain a lot larger than the number of parameters, and in all cases the associations exhibited very low significance level. Furthermore, even when all types of emergency presentations are studied, there is limited evidence for association [32].

Finally, anonymised GPPS information does not allow us to study the experience of patients who by-pass their GPs compared with those who do not. Moreover, we do not know the extent to which communication barriers between GPs and secondary care; the wish to expedite diagnosis, particularly in patients presenting at a later stage; the lack of clear 'appropriateness' guidelines, or other factors, drive GPs to send patients directly to emergency departments.

\section{Conclusion}

Despite the high incidence of lung cancer, primary care practices see few to very few lung cancer patients, which leads to high instability [41]. A large number of practices 
are susceptible to reach high proportions of lung cancer EP. High proportions of EP is a system-wide issue rather than a distinctive feature of practices exhibiting certain characteristics.

\section{Additional files}

Additional file 1: Table S1. Description of practice level characteristics

Additional file 2: Table S2. Characteristics of lung cancer patients diagnosed in 2006 to 2013 by emergency presentation type and for nonemergency presentations (DOCX 36 kb)

Additional file 3: Table S3. Comparison of logistic models and mixed logistic models for the modelling of emergency presentation by patient and practice variables, lung cancer patients diagnosed in 2010 (DOCX 19 kb)

Additional file 4: Table S4. OR for the models presented in Additional file 2: Table S2 and Additional file 5: Figure S1 (DOCX 48 kb)

Additional file 5: Figure S1. ROC curves associated with two sets of models defined in Additional file 1: Table S1. (DOCX $132 \mathrm{~kb}$ )

\section{Abbreviations}

A\&E: Accident and Emergency; AIC: Akaike Information Criterion; CAS: Cancer Analysis System; CCT: Cancer Commissioning Toolkit; COPD: Chronic Obstructive Pulmonary Disease; EP: Emergency Presentation; GP: General Practitioner; GPPS: GP Patient Survey; HES: Hospital Episode Statistics; IMD: Index for Multiple Deprivation; LSOA: Lower Super Output Area; LUCADA: Lung Cancer Audit Data; NCWT: National Cancer Waiting Times; NICE: National Institute for Health and Care Excellence; OR: Odds Ratio; PP: General Practice Profiles; QMAS: Quality Management and Analysis System; QOF: Quality Outcomes Framework; TNM: Tumour, Nodes, Metastasis; UK: United Kingdom; VPC: variance partition coefficient

\section{Funding}

CM and FJR participated in this research as part of the work of The Policy Research Unit in Cancer Awareness, Screening and Early Diagnosis. The Policy Research Unit in Cancer Awareness, Screening and Early Diagnosis receives funding for a research programme from the Department of Health Policy Research Programme. It is a collaboration between researchers from seven institutions (Queen Mary University of London, UCL, King's College London, London School of Hygiene and Tropical Medicine, Hull York Medical School, Durham University and Peninsula Medical School). BR was supported by Cancer Research UK grant number C7923/A18525. RR was (in part) supported by the National Institute for Health Research (NIHR) Collaboration for Leadership in Applied Health Research and Care (CLAHRC) North Thames at Bart's Health NHS Trust. The views expressed are those of the author(s) and not necessarily those of the NHS, the NIHR or the Department of Health. The findings and conclusions in this report are those of the authors.

\section{Availability of data and materials}

The cancer registry data that support the findings of this study are available from Public Health England, but restrictions apply to the availability of these data, which were used under license for the current study, and so are not publicly available. However dataset on General Practice characteristics are publicly available.

\section{Authors' contributions}

$R R$ had the original idea. BR, SWD and RR refined the project idea. BR led on the analyses. CM, NP and FJR conducted the analyses. GP provided expert advice on the Structural Equation Models. FJR provided expert advice and conducted the model selection analyses. CM, FJR, BR drafted the manuscript. All authors commented and amended drafts of the manuscript. All authors read and approved the final manuscript.

\section{Transparency declaration}

CM affirms that the manuscript is an honest, accurate, and transparent account of the study being reported; that no important aspects of the study have been omitted; and that any discrepancies from the study as planned (and, if relevant, registered) have been explained.

\section{Ethical approval and consent to participate}

We obtained the statutory approvals required for this research from the Confidentiality Advisory Group (CAG) of the Health Research Authority (HRA): PIAG 1-05(c)/2007; ECC 1-05(a)/2010, and the ethical approval were updated 6 April 2017 (REC 13/LO/0610) from the Research Ethics Committee (REC) of the Health Research Authority (HRA). We used previously collected data (National Cancer Registry data): No consent to participate was sought from patients.

\section{Competing interests}

The authors declare that they do not have any conflict of interest associated with this research, and the content is solely the responsibility of the authors. They declare no support from any organisation for the submitted work; no financial relationships with any organisations that might have an interest in the submitted work in the previous three years; no other relationships or activities that could appear to have influenced the submitted work.

\section{Publisher's Note}

Springer Nature remains neutral with regard to jurisdictional claims in published maps and institutional affiliations.

\section{Author details}

${ }^{1}$ Cancer Survival Group, London School of Hygiene and Tropical Medicine, Keppel street, London WC1E 7HT, UK. ${ }^{2}$ University College London, Department of Applied Health Research, London, UK. ${ }^{3}$ Centre for Longitudinal Studies, Department of Social Science, UCL - Institute of Education, University College London, London, UK. ${ }^{4}$ Queen Mary University of London, Wolfson Institute of Preventive Medicine, Centre for Cancer Prevention, London, UK.

Received: 25 January 2018 Accepted: 2 May 2018

Published online: 31 May 2018

\section{References}

1. Wilcock A, Crosby V, Hussain A, McKeever TM, Manderson C, Farnan S, et al. Lung cancer diagnosed following an emergency admission: mixed methods study of the management, outcomes and needs and experiences of patients and carers. Respir Med. 2016:5(114):38-45.

2. National Cancer Intelligence Network. In: Network NCl, editor. Routes to diagnosis - NCIN data briefing. London: Public Health England; 2010.

3. Porta M, Fernandez E, Belloc J, Malats N, Gallen M, Alonso J. Emergency admission for cancer: a matter of survival? Br J Cancer. 1998;77(3):477-84.

4. McPhail S, Elliss-Brookes L, Shelton J, Ives A, Greenslade M, Vernon S, et al. Emergency presentation of cancer and short-term mortality. Br J Cancer. 2013;109(8):2027-34.

5. Department of Health. Cancer Reform Strategy: Equality Impact Assessment 2007. Available from: http://webarchive.nationalarchives.gov.uk/ 20130104214031/http://www.dh.gov.uk/en/Publicationsandstatistics/ Publications/PublicationsLegislation/DH_081005. Accessed 8 May 2018.

6. The Independent Cancer Taskforce. Achieving world-class cancer outcomes: a strategy for England 2015-2020. 2015.

7. Hansen RP, Olesen F, Sorensen HT, Sokolowski I, Sondergaard J. Socioeconomic patient characteristics predict delay in cancer diagnosis: a Danish cohort study. BMC Health Serv Res. 2008;8:49.

8. Walter F, Webster A, Scott S, Emery J. The Andersen model of Total patient delay: a systematic review of its application in cancer diagnosis. J Health Serv Res Policy. 2012;17(2):110-8.

9. Andersen RS, Vedsted P, Olesen F, Bro F, Sondergaard J. Does the organizational structure of health care systems influence care-seeking decisions? A qualitative analysis of Danish cancer patients' reflections on care-seeking. Scand J Prim Health Care. 2011;29(3):144-9.

10. Coleman MP, Forman D, Bryant H, Butler J, Rachet B, Maringe C, et al. Cancer survival in Australia, Canada, Denmark, Norway, Sweden, and the UK, 1995-2007 (the international Cancer benchmarking partnership): an analysis of population-based cancer registry data. Lancet. 2011;377:127-38.

11. Office for National Statistics. Deaths registered in England and Wales (series DR): 2015. London: HMSO; 2016.

12. Exarchakou A, Rachet B, Nash E, Bannister N, Coleman MP, Rowlands S. Cancer survival in England: adults diagnosed in 2009 to 2013, followed up to 2014: Office for National Statistics; Newport, 2015. 
13. National Institute for Health and Clinical Excellence. Lung Cancer - the diagnosis and treatment of lung cancer: National Institute for Health and Clinical Excellence (NICE); 2011. Report No. 121.

14. Barrett J, Hamilton W. Pathways to the diagnosis of lung cancer in the UK: a cohort study. BMC Fam Pract. 2008;9:31.

15. Raine R, Wong W, Scholes S, Ashton C, Obichere A, Ambler G. Social variations in access to hospital care for patients with colorectal, breast, and lung cancer between 1999 and 2006: retrospective analysis of hospital episode statistics. BMJ. 2010;340:b5479.

16. Public Health England. Routes to diagnosis 2015 update: Lung Cancer. 2016; www.ncin.org.uk/view?rid=3120. Accessed 08 May 2018.

17. Abel GA, Shelton J, Johnson S, Elliss-Brookes L, Lyratzopoulos G. Cancer-specific variation in emergency presentation by sex, age and deprivation across 27 common and rarer cancers. Br J Cancer. 2015;112(Suppl 1):S129-36.

18. Rich AL, Tata LJ, Stanley RA, Free CM, Peake MD, Baldwin DR, et al. Lung cancer in England: information from the National Lung Cancer Audit (LUCADA). Lung Cancer. 2011;72(1):16-22.

19. Rubin G, Neal R, Abel G, Lyratzopoulos G. Integrated research efforts are needed to better understand how to reduce the proportion of patients with cancer who are diagnosed as emergencies. Br J Cancer. 2013;108(7):1550-1.

20. Benitez-Majano S, Fowler H, Maringe C, Di Girolamo C, Rachet B. Deriving stage at diagnosis from multiple population-based sources: colorectal and lung cancer in England. Br J Cancer. 2016;115(3):391-400. 06/21/online

21. Elliss-Brookes L, McPhail S, Ives A, Greenslade M, Shelton J, Hiom S, et al. Routes to diagnosis for cancer - determining the patient journey using multiple routine data sets. Br J Cancer. 2012;107(8):1220-6.

22. National Health and Social Care Information Center. NHS Outcome Framework indicators. 2010-11. https:/digital.nhs.uk/data-and-information/data-collectionsand-data-sets/data-collections/quality-and-outcomes-framework-qof.

23. The GP Patient Survey. IPSOS/MORI. http://www.gp-patient.co.uk/. Accessed 8 May 2018.

24. National Cancer Intelligence Network. General practice profiles for cancer. 2010. Available at http://www.ncin.org.uk/cancer_information_tools/profiles/ gp_profiles. Accessed 8 May 2018.

25. Spiegelhalter DJ. Funnel plots for comparing institutional performance. Stat Med. 2005;24(8):1185-202.

26. Kline RB. Principles and practice of structural equation modeling. 4th: Guilford Press; 2015. p. 534

27. Friedman $J H$, Hastie T, Tibshirani R. Regularization paths for generalized Linear models via coordinate descent. J Stat Softw. 2010;33(1):1-22.

28. Mathers N, Hodgkin P. The gatekeeper and the wizard: a fairy tale. BMJ. 1989;298(6667):172-4.

29. Lyratzopoulos G, Wardle J, Rubin G. Rethinking diagnostic delay in cancer: how difficult is the diagnosis? BMJ. 2014;349:97400.

30. Cowling TE, Harris M, Watt H, Soljak M, Richards E, Gunning E, et al. Access to primary care and the route of emergency admission to hospital: retrospective analysis of national hospital administrative data. BMJ Qual Saf. 2016;25(6):432-40.

31. Rogers S, Gildea C, Meechan D, Baker R. Access, continuity of care and consultation quality: which best predicts urgent cancer referrals from general practice? J Public Health. 2014;36(4):658-66.

32. Bankart MJ, Baker R, Rashid A, Habiba M, Banerjee J, Hsu R, et al. Characteristics of general practices associated with emergency admission rates to hospital: a cross-sectional study. Emerg Med J. 2011;28(7):558-63.

33. Mitchell ED, Pickwell-Smith B, Macleod U. Risk factors for emergency presentation with lung and colorectal cancers: a systematic review. BMJ Open. 2015;2015:5(4)

34. Oldale MJ, McKenna M, Waddecar S, Scott NA. Emergency admission with colorectal cancer: its relationship to primary health care organization. Color Dis. 2000;2(4):203-6.

35. Zhou Y, Abel GA, Hamilton W, Pritchard-Jones K, Gross CP, Walter FM, et al. Diagnosis of cancer as an emergency: a critical review of current evidence. Nat Rev Clin Oncol. 2017;14(1):45-56.

36. Rose PW, Rubin G, Perera-Salazar R, Almberg SS, Barisic A, Dawes M, et al. Explaining variation in cancer survival between 11 jurisdictions in the international Cancer benchmarking partnership: a primary care vignette survey. BMJ Open. 2015;5:5.

37. Niksic M, Rachet B, Warburton FG, Wardle J, Ramirez AJ, Forbes LJ. Cancer symptom awareness and barriers to symptomatic presentation in Englandare we clear on cancer? Br J Cancer. 2015;113(3):533-42.
38. Black G, Sheringham J, Spencer-Hughes V, Ridge M, Lyons M, Williams C, et al. Patients' experiences of Cancer diagnosis as a result of an emergency presentation: a qualitative study. PLoS One. 2015;10(8):e0135027.

39. Mitchell ED, Rubin G, Merriman L, Macleod U. The role of primary care in cancer diagnosis via emergency presentation: qualitative synthesis of significant event reports. Br J Cancer. 2015;112(Suppl 1):S50-S6.

40. Renzi C, Lyratzopoulos G, Card T, Chu TPC, Macleod U, Rachet B. Do colorectal cancer patients diagnosed as an emergency differ from nonemergency patients in their consultation patterns and symptoms[quest] a longitudinal data-linkage study in England. Br J Cancer. 2016;115(7):866-75.

41. Abel G, Saunders CL, Mendonca SC, Gildea C, McPhail S, Lyratzopoulos G. Variation and statistical reliability of publicly reported primary care diagnostic activity indicators for cancer: a cross-sectional ecological study of routine data. BMJ Qual Saf. 2017;28

\section{Ready to submit your research? Choose BMC and benefit from:}

- fast, convenient online submission

- thorough peer review by experienced researchers in your field

- rapid publication on acceptance

- support for research data, including large and complex data types

- gold Open Access which fosters wider collaboration and increased citations

- maximum visibility for your research: over $100 \mathrm{M}$ website views per year

At BMC, research is always in progress.

Learn more biomedcentral.com/submissions 